\title{
Desempenho Reprodutivo, Concentrações de Progesterona e Metabólitos Lipídicos no Pós-parto de Vacas Mestiças H/Z, Submetidas a uma Dieta Hiperlipidêmica ${ }^{1}$
}

\author{
José Antonio Delazari' ${ }^{2}$, Francisco Aloízio Fonseca ${ }^{3}$, Augusto Cézar de Queiroz ${ }^{4}$, José Carlos \\ Pereira $^{4}$, Paulo Roberto Cecon ${ }^{5}$
}

\begin{abstract}
RESUMO - Para avaliar o efeito de uma dieta hiperlipidêmica nos níveis séricos de metabólitos lipídicos e progesterona e no desempenho reprodutivo, 42 vacas mestiças foram submetidas a dois tratamentos: $\mathrm{T} 1$ ( $\mathrm{n}=21$ ) dieta-controle e T2 ( $\mathrm{n}=21)$ dieta hiperlipidêmica, tendo como principal fonte de lipídios o grão de soja integral. Os intervalos médios para a primeira e segunda ovulações pós-parto foram 26,3 e 35,9 dias para os animais do T1 e 21,7 e 37,4 dias para os do T2. Os intervalos médios do parto ao início da atividade luteal, primeiro estro e primeiro ciclo estral normal foram, respectivamente, 29,9; 39,5; e 53,9 dias para T1 e 25,7; 33,3; e 52,3 dias para T2. A duração média do primeiro ciclo estral foi de 14,7 e 16,9 dias e do segundo de 19,8 e 19,5 dias para T1 e T2, respectivamente. As concentrações de progesterona nos ciclos estrais normais ocorridos durante o período de 90 dias pós-parto variaram de valores mínimos de 0,27 (T1) e $0,31 \mathrm{ng} / \mathrm{mL}$ (T2), nos dias 0 e 1 (dia 0 = ovulação), para valores máximos de $6,71 \mathrm{ng} / \mathrm{mL}$ (T1), nos dias 10 e 11, e 7,04 ng/mL (T2), nos dias -9 e -8, retornando a níveis basais $(<1 \mathrm{ng} / \mathrm{mL})$ nos dias -3 e -2 . As concentrações médias de colesterol total e colesterol HDL para $\mathrm{T}_{1}$ e $\mathrm{T}_{2}$ foram, respectivamente, 100,74 e 67,3 mg/dL e 162,25 e 95,8 $\mathrm{mg} / \mathrm{dL}$. O efeito de hipercolesterolemia da dieta foi confirmado nesta pesquisa, mas sem nenhum aumento nas concentrações de progesterona e desempenho reprodutivo.
\end{abstract}

Palavras-chave: colesterol, dieta hiperlipidêmica, HDL, progesterona, vacas pós-parto

\section{Reproductive Performance, Concentrations of Progesterone and Lipidic Metabolites in the Postpartum Crossbred Dairy Cows Submitted to a Hyperlipidemic Diet}

\begin{abstract}
To evaluate the effect of a hiperlipidemic diet on the reproductive performance, lipidic metabolites and serum progesterone levels, 42 Holstein-Zebu crossbred cows were submitted to two treatments: T1 ( $\mathrm{n}=21)$, control diet, and T2 ( $\mathrm{n}=21$ ), hyperlipidemic diet, consisting mainly on whole soybean. The mean intervals to the first and second postpartum ovulations were 26.3 and 35.9 days for animals of T1 and 21.7 and 37.4 days for those of T2. The average intervals from calving to onset of the luteal activity, first estrus and first normal estrus cycle length were respectively, 29.9, 39.5, and 53.9 days for T1 and 25.7, 33.3, and 52.3 days for T2. The average first estrus cycle was 14.7 and 16.9 days and of the second 19.8 and 19.5 days for T1 and T2, respectively. The concentrations of serum progesterone in the normal estrus cycles during the period of 90 days postpartum, varied from minimum values of $0.27 \mathrm{ng} / \mathrm{mL}$ (T1) to $0.31 \mathrm{ng} / \mathrm{mL}$ (T2) on the days 1 and 0 (day $0=$ ovulation) to maximum values of 6.71 $\mathrm{ng} / \mathrm{mL}$ (T1) on days 10 and 11 and $7.04 \mathrm{ng} / \mathrm{mL}$ (T2) on days -9 and -8 , returning to basal levels $(<1 \mathrm{ng} / \mathrm{mL})$ on days -3 e -2 . The total cholesterol and HDL average concentrations for T1 e T2 were 100.74 and $67.3 \mathrm{mg} / \mathrm{dL}$ (T1) and $162.25 \mathrm{and} 95.8 \mathrm{mg} / \mathrm{dL}$ (T2) respectively. The hypercholesterolemic effect of the diet was confirmed, but without any increase in the concentration of progesterone and reproductive performance.
\end{abstract}

Key Words: cholesterol, hyperlipidemic diet, HDL, progesterone, postpartum cows

\section{Introdução}

Um período crítico e de grande importância em um sistema de produção de leite corresponde ao pósparto, quando normalmente ocorre desequilíbrio entre o requerimento do animal e a ingestão de nutrientes. Vacas em início de lactação podem apresentar situação de balanço energético negativo, haja vista a alta produção de leite, acompanhada de inadequada ingestão de alimentos.

Falhas no manejo alimentar neste período acarretam grandes prejuízos, pois baixo nível nutricional geralmente reduz a porcentagem de inseminações efetivas e, conseqüentemente, prolonga o intervalo

\footnotetext{
${ }^{1}$ Parte da tese apresentada pelo primeiro autor à Universidade Federal de Viçosa, para obtenção do título de "Magister Scientiae". Projeto financiado pela FAPEMIG/CNPq.

2 Médico Veterinário da EMATER-RIO.

3 Professor da UENF - Laboratório de Zootecnia e Nutrição Animal.

${ }^{4}$ Professor do Departamento de Zootecnia da UFV.

5 Professor do Departamento de Matemática da UFV .
} 
parto concepção (FOLMAN et al., 1973). SHORT e ADAMS (1988) relatam que nutrição deficiente provoca distúrbios reprodutivos por alteração nos mecanismos neuro-endócrinos e metabólicos, que levam à inatividade ovariana. Por outro lado, o restabelecimento precoce da função cíclica de ovário, estro e gestação é essencial para a manutenção de intervalo de partos satisfatório (STEVENSON e CALL, 1983).

Considerando a incapacidade dos animais em suprir suas exigências nutricionais na fase inicial do pós-parto, faz-se necessário o desenvolvimento de formas alternativas de alimentação, procurando melhorar a qualidade da dieta, com a utilização de produtos (alimentos) capazes de equilibrar ou mesmo atenuar o déficit nutricional pós-parto, com conseqüente melhoria no desempenho reprodutivo.

Regimes estratégicos de alimentação de vacas, incluindo os lipídios, têm sido usados como métodos para aliviar parte do déficit energético da dieta no período pós-parto (BAUMAN e CURRIE, 1980) e estimular a função ovariana (LUCY et al., 1992b).

Vários pesquisadores (PARK et al., 1983; TALAVERA et al., 1985; WILLIAMS, 1989; WEHRMAN et al., 1991; e HAWKINS et al., 1995) sugerem que o fornecimento de dietas hiperlipidêmicas pode incrementar a biossíntese luteal de progesterona, pela elevação dos níveis plasmáticos de colesterol. A suplementação de gorduras para vacas durante o pós-parto aumentou as concentrações de progesterona após a primeira ovulação (HIGHTSHOE et al.,1991), bem como em $18 \%$ a taxa de vacas com atividade luteal no início do pós-parto (WEHRMAN et al., 1991). Estudos recentes sugerem que o aumento no desenvolvimento folicular ovariano e na função luteal de vacas suplementadas com gordura deve-se às mudanças nas concentrações de metabólitos lipídicos e de hormônios metabólicos no soro, que podem agir no eixo hipotalâmico-hipofisário-ovariano (LUCY et al., 1992c; THOMAS e WILLIAMS, 1996). Por outro lado, HAWKINS et al. (1995) reportaram que vacas suplementadas com gorduras apresentaram menor taxa de desaparecimento de progesterona do soro sangüíneo.

A fertilidade em vacas leiteiras foi maior quando as concentrações de progesterona foram mais altas no leite, de 7 a 4 dias antes da inseminação (MEISTERLING e DAILEY, 1987), ou no sangue, durante 12 dias antes da inseminação (FONSECA et al., 1983). Dados com vacas leiteiras sincronizadas tanto com PGF- $2 \alpha$ apenas ou associada a progestágeno indicaram que concentrações préovulatórias de progesterona foram positivamente correlacionadas com a fertilidade (BRITT et al., 1994). Por isso, o efeito da alimentação com dietas hiperlipidêmicas durante o pós-parto de vacas deve ser pesquisado nas condições de manejo dos rebanhos mestiço $\mathrm{H} / \mathrm{Z}$, para que se possam estabelecer estratégias nutricionais com o objetivo de melhorar o desempenho reprodutivo (GRUMMER e CARROLL, 1988).

Considerando a hipótese de que o lipídio por si só, e não a energia adicional das dietas hiperlipidêmicas, estimula o ovário e aumenta o desenvolvimento folicular (LUCY et al., 1992a), objetivou-se, no presente experimento, verificar, com o uso de rações isoenergéticas e isoprotéicas, a ação efetiva de uma dieta hiperlipidêmica no desempenho reprodutivo de vacas leiteiras H/Z no início do pós-parto.

\section{Material e Métodos}

O experimento foi conduzido no setor de bovinocultura de leite do Departamento de Zootecnia da Universidade Federal de Viçosa. Foram utilizadas 42 vacas leiteiras mestiças $\mathrm{H} / \mathrm{Z}$, distribuídas em dois grupos de tratamento: $\mathrm{T} 1(\mathrm{n}=21)$ dieta controle, na qual foi utilizado um suplemento concentrado já adotado no sistema de produção de leite da Universidade, e T2 $(n=21)$ dieta hiperlipidêmica, que teve como principal fonte de lipídios o grão de soja integral. Os animais foram distribuídos em cada grupo a partir do dia do parto, continuando por 90 dias. Os suplementos foram formulados para serem isoenergéticos e isoprotéicos com $72,6 \%$ de NDT e $21,8 \%$ de proteína bruta na matéria seca, diferindo apenas nos níveis de extrato etéreo com $2,56 \%$ para o suplemento do $\mathrm{T} 1 \mathrm{e}$ 9,56\% para o do T2. As análises laboratoriais dos alimentos, realizadas posteriormente, forneceram valores de 21,41e 22,61\% de proteína bruta e 2,65 e 10,28\% de extrato etéreo, respectivamente, para os suplementos do T1 e do T2 Na ração total (volumoso + concentrado), o nível de extrato etéreo foi de 2,50 e 6,34\% para $\mathrm{T} 1$ e T2 Os primeiros sete dias pós-parto foram de adaptaçã̃o dos animais ao manejo e às rações.

Todos os animais receberam, como volumoso, silagem de milho, que foi misturada ao suplemento concentrado, para disponibilidade contínua dos nutrientes.

A proporção dos ingredientes nos suplementos é apresentada na Tabela 1.

Quinze dias após o parto, todas as vacas foram 
Rev. bras. zootec.

Tabela 1 - Proporção dos ingredientes usados nos suplementos (\% matéria natural)

Table 1 - Proportion of ingredientes used in the supplements (\% fresh matter basis)

\begin{tabular}{|c|c|c|}
\hline \multirow[t]{2}{*}{$\begin{array}{l}\text { Ingrediente } \\
\text { Ingredient }\end{array}$} & \multicolumn{2}{|c|}{$\begin{array}{c}\text { Tratamento } \\
\text { Treatment }\end{array}$} \\
\hline & $\mathrm{T} 1$ & $\mathrm{~T} 2$ \\
\hline Fubá & 59,98 & 47,30 \\
\hline $\begin{array}{l}\text { Corn meal } \\
\text { Farelo de soja } \\
\text { Sovbean meja }\end{array}$ & 37,00 & - \\
\hline $\begin{array}{l}\text { Soybean meal } \\
\text { Soja grão }\end{array}$ & - & 45,00 \\
\hline $\begin{array}{l}\text { Soybean seed } \\
\text { Farinha de ossos } \\
\text { Bone meal }\end{array}$ & 1,00 & 1,00 \\
\hline $\begin{array}{l}\text { Calcário } \\
\text { Limestone }\end{array}$ & 1,00 & 1,00 \\
\hline $\begin{array}{l}\text { Sal } \\
\text { Salt }\end{array}$ & 1,00 & 1,00 \\
\hline $\begin{array}{l}\text { Premix } \\
\text { Veículo inerte }\end{array}$ & 0,02 & $\begin{array}{l}0,02 \\
468\end{array}$ \\
\hline $\begin{array}{l}\text { Veículo inerte } \\
\text { Inert vehicle }\end{array}$ & & \\
\hline
\end{tabular}

submetidas a um exame ginecológico para avaliar o processo de involução uterina, verificar a presença de estruturas nos ovários e detectar anomalias e/ou patologias dos órgão genitais. Animais com problema foram descartados da pesquisa. Posteriormente, em intervalos semanais, foram realizadas palpações retais para verificação das condições do útero (diâmetro e tônus) e dos ovários (tamanho, presença de folículos e/ou corpos lúteos). As vacas foram mantidas isoladas de rufiões e fêmeas androgenizadas, para evitar que a exposição dos animais à bioestimulação (ZALESKY et al., 1984; BURNS e SPITZER, 1992) pudesse talvez mascarar algum efeito da dieta hiperlipidêmica no desempenho reprodutivo pósparto (CARR et al., 1994).

Os sinais comportamentais de estro foram observados ao amanhecer, ao escurecer e nos horários de trato dos animais (manhã e tarde). O dia de cada ovulação foi estimado pela palpação retal, pelos dados do estro e pelo perfil de progesterona, seguindo critério adotado por FONSECA et al. (1983).

As concentrações de progesterona foram agrupadas em períodos de dois dias do ciclo estral. Para ajustar as variações na duração dos ciclos estrais das vacas, os dados foram padronizados para ciclos de 21 dias (HELMER e BRITT, 1986; RIBADU et al., 1994), de acordo com o seguinte critério: o ciclo estral foi representado por dias seguintes à ovulação $(0$ a 11) e dias que precederam a ovulação subseqüente ( -9 a 0 ).

Amostras de sangue foram coletadas duas vezes por semana, com intervalos de três a quatro dias, por punção dos vasos coccígeos medianos, utilizando o sistema de colheita a vácuo (vacutainer) sem anticoagulante. Estas amostras foram centrifugadas dentro de no máximo uma hora da coleta durante 30 minutos a $1699 \mathrm{~g}$. O soro obtido foi transferido para vidros esterilizados com tampa, identificados e armazenados a $-19^{\circ} \mathrm{C}$. Foram utilizados testes enzimáticos (bio Mérieux) para determinar as concentrações sangüíneas de metabólitos lipídicos, e imunoenzimáticos (Progestérone EIA-bio Mérieux) para traçar o perfil de progesterona no soro .

As análises químico-bromatológicas das amostras de silagem, concentrados e sobras foram realizadas conforme SILVA (1990), para avaliar teores de matéria seca, matéria orgânica, extrato etéreo, fibra em detergente neutro e proteína bruta.

A composição química dos suplementos concentrados e da silagem de milho é apresentada na Tabela 2.

Os resultados foram interpretados por meio de análise de variância e de regressão. As médias dos fatores qualitativos foram comparadas pelo teste $\mathrm{F}$. Para interpretar as concentrações médias de progesterona nos tratamentos $\mathrm{T} 1$ e $\mathrm{T} 2$, em função dos dias do ciclo estral, foi ajustada uma equação de regressão para cada tratamento (Tabela 3). A técnica de identidades de modelos foi usada para verificar a existência ou não de diferenças nas concentrações do hormônio entre T1 e T2.

\section{Resultados e Discussão}

Três vacas apresentaram patologias durante o experimento. Uma vaca do $\mathrm{T} 1 \mathrm{e}$ outra do $\mathrm{T} 2$ apresentaram corpo lúteo persistente, seguido de anestro e manutenção de níveis elevados de progesterona, a partir da segunda ovulação. Uma vaca do T2 desenvolveu cisto folicular.

Nenhuma das variáveis reprodutivas examinadas no presente estudo (Tabela 4$)$ diferiu $(\mathrm{P}>0,05)$ entre os dois tratamentos. Estes resultados são consonantes com os obtidos por RUEGSEGGER e SCHULTZ (1985), que também utilizaram grão de soja integral como fonte de lipídios para vacas leiteiras, em início de lactação, e não observaram efeito de dieta no número de dias para primeiro estro, primeira inseminação e taxa de concepção. Falhas de dietas hiperlipidêmicas em afetar características reprodutivas também foram relatadas em vacas de leite (SCHINGOETHE e CASPER, 1991) e de corte (CARR et al., 1994; LAMMOGLIA et al., 1996), 
Tabela 2 - Teor de matéria seca (MS), proteína bruta (PB), extrato etéreo (EE), fibra em detergente neutro (FDN), cinzas e matéria orgânica (MO) (\% MS) nos suplementos concentrados e na silagem de milho

Table 2 - Content of dry matter (DM), crude protein $(C P)$, ether extract (EE), neutral detergent fiber (NDF), ashes, and organic matter (OM) (\%DM) in the concentrate supplements and corn silage

\begin{tabular}{|c|c|c|c|c|c|c|}
\hline \multirow{3}{*}{$\begin{array}{l}\text { Dieta } \\
\text { Diet }\end{array}$} & \multicolumn{6}{|c|}{$\begin{array}{l}\text { Nutriente } \\
\text { Nutrient }\end{array}$} \\
\hline & MS & PB & $\mathrm{EE}$ & FDN & Cinzas & MO \\
\hline & $D M$ & $C P$ & & $N D F$ & Ash & $O M$ \\
\hline $\begin{array}{l}\text { Suplemento-controle } \\
\text { Control supplement }\end{array}$ & 87,48 & 21,41 & 2,65 & 21,80 & 4,25 & 95,75 \\
\hline $\begin{array}{l}\text { Suplementohiperlipidêmico } \\
\text { Hyperlipidemic supplement }\end{array}$ & 88,45 & 22,61 & 10,28 & 24,57 & 3,95 & 96,05 \\
\hline $\begin{array}{l}\text { Silagem de milho } \\
\text { Corn silage }\end{array}$ & 31,58 & 6,63 & 2,35 & 62,14 & 4,51 & 95,46 \\
\hline
\end{tabular}

Tabela 3 - Regressão ajustada, para comparação dos valores médios das concentrações séricas de progesterona, em função dos dias dos ciclos estrais normais ocorridos durante 90 dias pós-parto

Table 3 - Adjusted regression for comparison of average values of the progesterone serum concentrations on days of normal estrus cycles during 90 days postpartum

\begin{tabular}{lccr}
\hline $\begin{array}{l}\text { Tratamento } \\
\text { Treatment }\end{array}$ & $\begin{array}{c}\text { Regressão } \\
\text { Regression }\end{array}$ & $\mathrm{R}^{2}$ & $\mathrm{~N}$ \\
\hline $\mathrm{T} 1$ & $\hat{\mathrm{Y}}=-2,13596+1,40396 \mathrm{D}-0,0626224 \mathrm{D}^{2}$ & 0,8887 & 11 \\
$\mathrm{~T} 2$ & $\hat{\mathrm{Y}}=-2,36854+1,52952 \mathrm{D}-0,0676952 \mathrm{D}^{2}$ & 0,8784 & 11 \\
Comum & $\hat{\mathrm{Y}}=-2,25225+1,46674 \mathrm{D}-0,0651588 \mathrm{D}^{2}$ & 0,8775 & 22 \\
Common & & &
\end{tabular}

mas outros têm reportado influências positivas de suplementação de gordura na função reprodutiva no pós-parto (WEHRMAN et al., 1991). Por outro lado, SCHNEIDER et al. (1988), utilizando ácidos graxos complexados a sais de cálcio, obtiveram taxa de concepção ao primeiro serviço de 60,5 vs $43,1 \%$ para vacas da dieta-controle.

O intervalo médio do parto à primeira ovulação de 23,9 dias, estimado neste estudo, é consistente com valores anteriormente relatados, de 23,6 dias por BRITT et al. (1974); 20,8 dias por FONSECA et al. (1983); 23,9 dias por VILLA-GODOY et. al. (1988); e 21,9 dias por STAPLES et al. (1990).

Das 39 vacas que ovularam durante o período experimental, apenas seis manifestaram estro antes da primeira ovulação (15\%). STEVENSON e CALL (1983) detectaram estro antes da primeira ovulação, em $24 \%$ das vacas. A ausência ou a expressão sub normal do estro, antes da primeira ovulação pós parto, assim como a subsequiente redução da fase luteal, sugere que, nestas situações, os mecanismos de balanço endócrino são incapazes de estabelecer ciclos estrais normais (CALLAHAN et al., 1971).

O intervalo médio estimado do parto ao início da atividade luteal foi de 29,9 \pm 16,3 e 25,7 \pm 7,6 dias para $\mathrm{T} 1$ e $\mathrm{T} 2$, respectivamente $(\mathrm{P}>0,05)$. Embora não tenham observado efeito de suplementação lipídica na recuperação da atividade ovariana pós-parto, SKLAN et al. (1991) observaram melhoria significativa na taxa de concepção do segundo serviço e diminuição no intervalo parto-prenhez.

O intervalo médio estimado do parto ao primeiro estro de 36,7 dias foi similar aos 34 dias, verificado por CALLAHAN et al. (1971), e 33,6 dias por DEL CARLO et al. (1987).

A duração do primeiro ciclo estral variou entre 6 e 28 dias. De maneira similar, FONSECA et al. (1983) constataram variação de 9 a 28 dias e STAPLES et al. (1990), de 6 a 22 dias.

Em ambos os tratamentos, ocorreram ciclos estrais de curta duração após a primeira ovulação $(14,7 \pm 6,2$ e 16,9 $\pm 6,9$ dias para T1 e T2, respectivamente). Entretanto, tem sido sugerido que dietas hiperlipidêmicas possuem o potencial de aumentar a ocorrência de corpo 
Tabela 4 - Média e desvio-padrão de características do desempenho reprodutivo de vacas mestiças $H / Z$, submetidas a duas dietas $(T 1=$ controle e $T 2=$ hiperlipidêmica), no período de 90 dias pós-parto

Table 4 - Mean and standard deviation of reproductive performance characteristics in crossbred dairy cows submitted to two diets $(T 1=$ control and $T 2=$ hyperlipidemic $)$, on the period of 90 days postpartum

\begin{tabular}{|c|c|c|c|}
\hline \multicolumn{4}{|c|}{ Dieta } \\
\hline \multirow[b]{2}{*}{$\begin{array}{l}\text { Variável } \\
\text { Variable }\end{array}$} & \multicolumn{2}{|c|}{ Diet } & \multirow[b]{2}{*}{$\begin{array}{l}\text { Média } \\
\text { Mean }\end{array}$} \\
\hline & $\mathrm{T} 1$ & $\mathrm{~T} 2$ & \\
\hline$\overline{1^{\mathrm{a}} \mathrm{OPP}^{1}}$ & $26,3 \pm 16,0(19)$ & $21,7 \pm 7,2(20)$ & $23,9 \pm 12,4(39)$ \\
\hline $2^{\mathrm{a}} \mathrm{OPP}^{2}$ & $35,9 \pm 7,7(17)$ & $37,4 \pm 10,5(17)$ & $36,7 \pm 9,1(34)$ \\
\hline $\mathrm{IPAL}^{3}$ & $29,9 \pm 16,3(19)$ & $25,7 \pm 7,6(20)$ & $27,7 \pm 12,6(39)$ \\
\hline $\mathrm{IPE}^{4}$ & $39,5 \pm 16,4(19)$ & $33,3+12,1(16)$ & $36,7 \pm 14,7(35)$ \\
\hline $\mathrm{IPCN}^{5}$ & $53,9 \pm 12,6(17)$ & $52,3 \pm 11,6(17)$ & $53,1 \pm 11,9(34)$ \\
\hline DPCE $^{6}$ & $14,7 \pm 6,2(19)$ & $16,9 \pm 6,9(19)$ & $15,8 \pm 6,6(38)$ \\
\hline $\mathrm{DSCE}^{7}$ & $19,8+3,1(16)$ & $19,5 \pm 4,5(16)$ & $19,7 \pm 3,8(32)$ \\
\hline $\mathrm{DPCN}^{8}$ & $20,2 \pm 1,8(17)$ & $20,2 \pm 1,8(18)$ & $20,2 \pm 1,8(35)$ \\
\hline
\end{tabular}

Valores entre parênteses correspondem ao número de animais.

1 Primeira ovulação pós-parto.

2 Segunda ovulação pós-parto.

3 Intervalo parto-início de atividade luteal.

4 Intervalo parto-primeiro estro.

5 Intervalo parto-primeiro ciclo estral normal (17 a 24 dias).

${ }_{7}^{6}$ Duração do primeiro ciclo estral.

7 Duração do segundo ciclo estral.

8 Duração do primeiro ciclo estral normal.

Values in parenthesis correspond to number of animals.

1 First postpartum ovulation.

2 Second postpartum ovulation.

3 Interval from calving to onset of the luteal activity.

4 Interval from calving to first oestrus.

5 Interval from calving to first normal oestrus cycle (17 to $24 d$ ).

6 Duration of first oestrus cycle.

7 Duration of second oestrus cycle.

8 Duration of first normal oestrus cycle.

lúteo com função normal, no período pós-parto. Utilizando vacas com ovulações induzidas por GnRH e alimentadas com dietas hiperlipidêmicas, WILLIAMS (1989) verificou que a duração média da primeira fase luteal pós-parto, de 15,3 dias, foi, aproximadamente, o dobro dos 7,2 dias das vacas, que receberam dieta-controle, sugerindo que dietas hiperlipidêmicas, durante o período pós-parto, podem reduzir a ocorrência de ciclos de curta duração.

A taxa de gestação foi de $53 \%$ para as 30 vacas inseminadas durante os 90 dias pós-parto. Das 12 vacas não-inseminadas, oito foram do T2 vs quatro do $\mathrm{T} 1$, das quais cinco do T2 e duas do T1 não apresentaram estro, durante o período experimental.

Na Tabela 5 e Figura 1, estão as concentrações médias de progesterona no soro sangüíneo das vacas dos tratamentos T1 e T2, para todos os ciclos estrais normais (17 a 24 dias) ocorridos durante os 90 dias pós-parto. Observa-se que, em 34 ciclos estrais normais de 17 vacas no T1 e em 33 ciclos estrais normais de 18 vacas do $\mathrm{T} 2$, as concentrações de progesterona no soro sangüíneo variaram de valores mínimos de

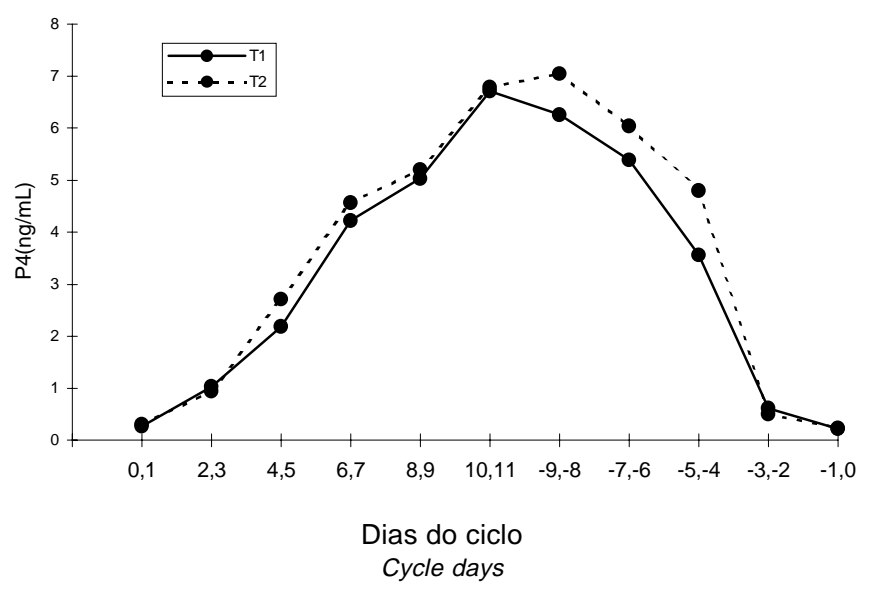

Figura 1 - Concentrações médias de progesterona (P4) no soro sangüíneo de vacas mestiças $H / Z$ submetidas a duas dietas $(\mathrm{T} 1=$ controle e $\mathrm{T} 2=$ hiperlipidêmica) nos ciclos estrais normais, ocorridos no período experimental.

Figura 1 - Average serum concentrations of progesterone (P4) of crossbred cows submitted to two diets $(T 1=$ control and $\mathrm{T} 2=$ hyperlipidemic) in the normal estrus cycle, ocurred in the experimental period. 
Tabela 5 - Concentrações médias de progesterona (ng/ $\mathrm{mL}$ ) no soro sangüíneo de vacas mesticas $\mathrm{H} / \mathrm{Z}$, submetidas a duas dietas ( $\mathrm{T} 1=$ controle e T2 = hiperlipidêmica) durante os ciclos estrais normais, ocorridos no período experimental (90 dias pós-parto)

Table 5 - Average serum progesterone $(\mathrm{ng} / \mathrm{mL})$ concentrations of crossbred cows submitted to two diet ( $T 1=$ control and $T 2$ = hyperlipidemic) during the normal estrus cycle, ocurred in the experimental period (90 days postpartum)

\begin{tabular}{llc}
\hline & \multicolumn{2}{c}{$\begin{array}{c}\text { Progesterona }(\mathrm{ng} / \mathrm{mL}) \\
\text { Progesterone }\end{array}$} \\
\cline { 2 - 3 } $\begin{array}{l}\text { Dias do ciclo estral } \\
\text { Days oestrus cycle }\end{array}$ & $\mathrm{T} 1$ & $\mathrm{~T} 2$ \\
\hline 0 e 1 & 0,27 & 0,31 \\
2 e 3 & 1,03 & 0,95 \\
4 e 5 & 2,18 & 2,71 \\
6 e 7 & 4,22 & 4,57 \\
8 e 9 & 5,03 & 5,20 \\
10 e 11 & 6,71 & 6,78 \\
-9 e -8 & 6,26 & 7,04 \\
-7 e -6 & 5,39 & 6,04 \\
-5 e -4 & 3,56 & 4,80 \\
-3 e -2 & 0,61 & 0,50 \\
-1 e 0 & 0,22 & 0,23 \\
\hline
\end{tabular}

${ }^{1}$ Dia $0=$ ovulação.

${ }^{1}$ Day $0=$ ovulation.

$0,27 \mathrm{ng} / \mathrm{mL}$ (T1) e $0,31 \mathrm{ng} / \mathrm{mL}$ (T2), nos dias 0 e 1 , até valores máximos de $6,71 \mathrm{ng} / \mathrm{mL}$ (T1) nos dias 10 e 11 e 7,04 ng/mL (T2), nos dias -9 e -8 , retornando a níveis basais $(<1 \mathrm{ng} / \mathrm{mL})$ nos dias -3 e -2 .

Estas concentrações podem ser consideradas normais e foram semelhantes às encontradas por STABENFELDT et al. (1969), DONALDSON et al. (1970), HENRICKS et al. (1971) e RIBADU et al. (1994).

A comparação das concentrações séricas de progesterona utilizando a técnica de identidade de modelos permitiu concluir que não houve diferença ( $\mathrm{P}>0,05)$ entre T1 e T2. Em estudo com novilhas mestiças H/Z e utilizando grão de soja integral como principal fonte de lipídio, OLIVEIRA (1995) também não encontrou aumento nas concentrações de progesterona para o grupo tratado com dieta hiperlipidêmica. Entretanto, outros estudos verificaram aumento nas concentrações de progesterona, durante o pós-parto de vacas tratadas com dieta hiperlipidêmica (CARROLL et al., 1990; SKLAN et al., 1991; e SPICER et al., 1993).

As concentrações médias semanais de colesterol total e colesterol HDL (Figuras 2 e 3), a partir da segunda semana pós-parto até a décima terceira, aumentaram, gradativamente, nos dois tratamentos, sendo que as concentrações médias, durante o perío-

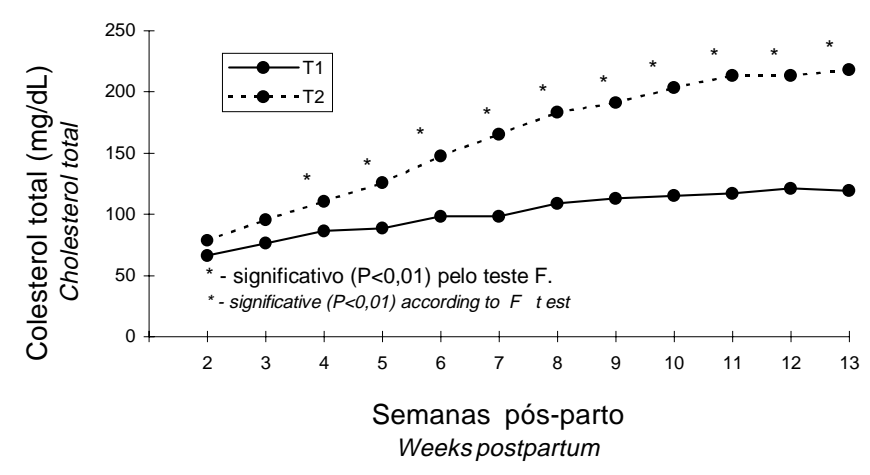

Figura 2 - Concentrações médias semanais de colesterol total ( $\mathrm{mg} / \mathrm{dL}$ ) no soro sanguíneo de vacas mestiças $H / Z$, submetidas a duas dietas ( $\mathrm{T} 1$ = controle e T2 = hiperlipidêmica) .

Figure 2 - Average weekly serum cholesterol concentrations of crossbred cows submitted to two diets ( $T 1=$ control and T2 = hyperlipidemic).

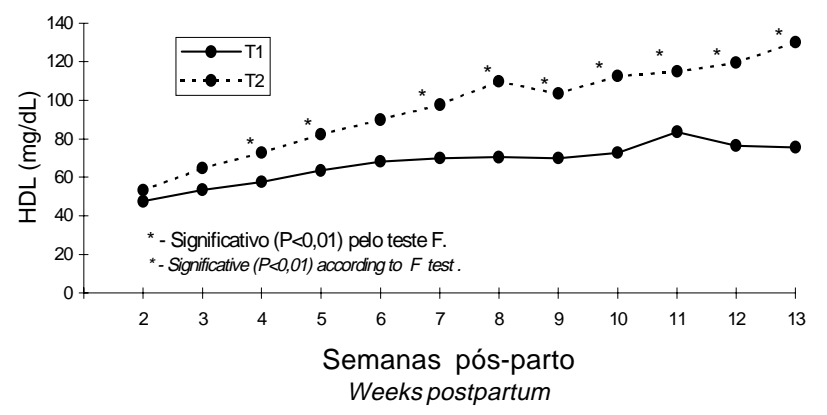

Figura 3 - Concentrações médias semanais de HDL (mg/ $\mathrm{dL}$ ) no soro sangüíneo de vacas mestiças $\mathrm{H} / \mathrm{Z}$, submetidas a duas dietas $(\mathrm{T} 1=$ controle $\mathrm{e}$ $\mathrm{T} 2$ = hiperlipidêmica).

Figure 3 - Average weekly serum $H D L(\mathrm{mg} / \mathrm{dL})$ concentrations of crossbred cows submitted to two diets ( $T 1=$ control and $T 2$ = hyperlipidemic) .

do avaliado, foram de 100,74 e $162,25 \mathrm{mg} / \mathrm{dL}$ de colesterol total e 67,3 e $95,8 \mathrm{mg} / \mathrm{dL}$ de colesterol HDL, para T1 e T2, respectivamente. Comportamento similar foi observado por KRONFELD et al. (1980), KWEON et al. (1986) e CARROLL et al. (1990).

Diferenças significativas $(\mathrm{p}<0,01)$ entre $\mathrm{T} 1$ e T2 foram observadas a partir da quarta semana pósparto, tanto para o colesterol total, como para o colesterol HDL, concordando com pesquisas anteriores, que também demonstraram que dietas com altos teores de lipídios aumentam o colesterol sangüíneo em bovinos (RAFALOWSKI e PARK, 1982; 
SCHAUFF et al., 1992; MÂNCIO, 1994; e OLIVEIRA, 1995). THOMAS e WILLIAMS (1996) encontraram concentrações séricas elevadas de colesterol total e colesterol HDL em animais suplementados com gordura, enquanto BAO et al. (1995) demonstraram que o colesterol HDL estimula a divisão celular e a produção de $I_{1} F_{1}$ em culturas de células da granulosa, o que possivelmente poderia ser um dos mecanismos pelos quais a gordura estaria afetando a reprodução de vacas no período pós-parto, mas não observado no presente estudo.

\section{Conclusões}

A dieta com alto teor de lipídios (grãos de soja integral) teve efeito de hipercolesterolemia, mas sem aumento nas concentrações séricas de progesterona e melhoria no desempenho reprodutivo dos animais.

\section{Referências Bibliográficas}

BAO, B., THOMAS, M.G., GRIFFITH, M.K. et al. 1995. Steroidogenic activity, insulin-like growth factor-1 production and proliferation of granulosa and theca cells obtained from dominant preovulatory and nonovulatory follicles during the bovine estrous cycle: Effects of lowdensity and high-density lipoproteins. Biol. Reprod, 53:12711279.

BAUMAN, D.E., CURRIE, W.B. 1980. Partitioning of nutrients during pregnancy and lactation: A review of mechanisms involving homeostasis and homeorhesis. J. Dairy Sci., 63:15141529.

BRITT, J.H., KITTOK, R.J., HARRISON, D.S. 1974. Ovulation, estrus and endocrine response after GnRH in early postpartum cows. J. Anim. Sci., 39:915-919.

BRITT, J.H., WASHBURN, S.P., FOUNTS, W.B. 1994. Reproductive performance of Holstein cows treated with prostaglandin-F2 $\alpha$ (PGF) and progesterone releasing intravaginal devices (PRID) in a controlled breeding program. J.Anim. Sci., 72:230(Suppl).

BURNS, P.D., SPITZER, J.C. 1992. Influence of biostimulation on reproduction in postpartum beef cows. J. Anim. Sci.,70:358362.

CALLAHAN, C.J., ERB, R.E., SURVE, A.H. et al. 1971. Variables influencing ovarian cycles in postpartum dairy cows. J. Anim Sci., 33:1053-1059.

CARR, D.L., SPITZER, J.C., JENKINS, T.C. et al. 1994. Effect of dietary lipid supplementation on progesterone concentration and reproductive performance in suckled beef cows. Theriogenology, 41:423-435.

CARROLL, D.J., JERRED, M.J., GRUMMER, R.R. et al. 1990. Effects of fat supplementation and immature alfafa to concentrate ratio on plasma progesterone, energy balance and reproductive traits of dairy cattle. J. Anim. Sci., 73:2855-2863.

DEL CARLO, R.J., ARAÚJO, R.B., PEREIRA, J.R.A. et al. 1987. Avaliação da atividade ovariana e fertilidade de vacas leiteiras, após parto normal. Rev. Bras. Med. Vet., 9:76-80.

DONALDSON, L.E., BASSETT, J.M., THORNBURN, G.D. 1970. Peripheral plasma progesterone concentration of cows during puberty, oestrus cycles, pregnancy and lactation and the effects of undernutrition or exogenous oxytocin on progesterone concentration. J. Endocrinology, 48:599-614.

FOLMAN, Y., ROSEMBERG, M., HERZ, Z. et al. 1973. The relationship between plasma progesterone concentration and conception in post-partum dairy cows maintained on two levels of nutrition. J. Reprod. Fert., 34:267-278.

FONSECA, F.A., BRITT, J.H., McDANIEL, B.T. et al. 1983. Reproductive traits of holsteins and Jerseys. Effects of age, milk yield, and clinical abnormalities on involution of cervix and uterus, ovulation, estrous cycles, detection of estrus, conception rate, and days open. J. Dairy Sci., 66:1128-1147.

GRUMMER, R.R., CARROLL, D.J. 1988. A review of lipoprotein cholesterol metabolism: Importance to ovarian function. J. Anim. Sci., 66:3160-3173.

HAWKINS, D.E., NISWENDER, K.D., OSS, G.M. et al. 1995. An increase in serum lipids increases luteal lipid content and alters the disappearance rate of progesterone in cows. J. Anim. Sci., 73:541-545.

HELMER, S.D., BRITT, J.H. 1986. Fertility of dairy cattle treated with human chorionic gonadotropin (hCG) to stimulate progesterone secretion. Theriogenology, 26:683-695.

HENRICKS, D.M., DICKEY, J.F., HILL, J.R. 1971. Plasma estrogen and progesterone levels in cows prior to and during estrus. Endocrinology, 89:1350-1355.

HIGHTSHOE, R.B., COCHRAN.R.C., CORAH, G.H. et al. 1991. Effects of calcium soaps of fatty acids on postpartum reproductive function in beef cows. J. Anim. Sci., 69:4097-4103.

KRONFELD, D.S., DONOGHUE, S., NAYLOR, J.M. et al. 1980. Metabolic effects of feeding protected tallow to dairy cows. J. Dairy Sci., 63:545-552.

KWEON, O.K., ONO, H., OSASA, K. et al. 1986. Factors affecting serum total cholesterol level of lactating holstein cows. Jpn J. Vet. Sci., 48:481-486.

LAMMOGLIA, M.A., WILLARD,S.T., OLDHAM, J.R. et al. 1996. Effects of dietary fat and season on steroid hormonal profiles before parturition and on hormonal, cholesterol, triglycerides, follicular patterns, and postpartum reproduction in Brahman cows. J. Anim. Sci., 74:2253-2262.

LUCY, M.C., SAVIO, J.D., BADINGA, L. et al. 1992a. Factors that affect ovarian follicular dynamics in cattle. J. Anim. Sci., 70:3615-3626.

LUCY, M.C., STAPLES, C.R., THATCHER, W.W. et al. 1992b. Influence of diet composition, dry-matter intake, milk production and energy balance on time of postpartum ovulation and fertility in dairy cows. Anim. Prod., 54:323-331.

LUCY, M.C.,THATCHER, W.W., STAPLES, C.R. 1992c. Postpartum function: nutricional and physiological interactions. In: Van HORN, H.H., WILCOX, C.J. (Eds.) Large Dairy Herd Management pp 135-145. American Dairy Science Association, Champaign, IL.

MÂNCIO, A.B. Plano nutricional, gonadotropina coriônica humana $(h C G)$ e amamentação na função reprodutiva $e$ metabólica de fêmeas bovinas: Viçosa, MG: UFV,1994. 158p. Tese (Doutorado em Zootecnia) - Universidade Federal de Viçosa, 1994.

MEISTERLING, E.M., DAILEY, R.A. 1987. Use of concentrations of progesterone and estradiol $17 \beta$ in milk in monitoring postpartum ovarian function in dairy cows. $J$. Dairy Sci., 70:2154-2161.

OLIVEIRA, F.N. Concentrações sangüíneas de progesterona e metabólitos lipídicos em novilhas tratadas com norgestomet e valerato de estradiol (Sincro-Mate B) e submetidas à dieta 
hiperlipidêmica. Viçosa, MG: UFV, 1995. 75p. Dissertação (Mestrado em Zootecnia)- Universidade Federal de Viçosa, 1995.

PARK, C.S., RAFALOWSKI, W., MARX, G.D. 1983. Effect of dietary fat supplement on lipid metabolism of holstein heifers. J. Dairy Sci., 66:528-534.

RAFALOWSKI, W. PARK, C.S. 1982. Whole sunflower seed as a fat supplement for lactating cows. J. Dairy Sci., 65:14841492.

RIBADU, A.Y., WARD, W.R., DOBSON, H. 1994.Comparative evaluation of ovarian structures in cattle by palpation per rectum, ultrasonography and plasma progesterone concentration. Vet. Rec., 135:452-457.

RUEGSEGGER, G.J., SCHULTZ. L.H. 1985. Response of high producing dairy cows in early lactation to the feeding of heattreated whole soybeans. J. Dairy Sci., 68:3272-3279.

SCHAUFF, D.J., ELLIOTT, J.P., CHARK, J.H. et al. 1992. Effects of feeding lactating dairy cows diets containing whole soybeans and tallow. J. Dairy Sci., 75:1923-1935.

SCHINGOETHE, D.J., CASPER, D.P. 1991. Total lactational response to added fat during early lactation. J. Dairy Sci., 74:2617-2622.

SCHNEIDER, P., SKLAN, D., CHALUPA, W. et al. 1988. Feeding calcium salts of fatty acids to lactating cows. J. Dairy Sci., 71:2143-2150.

SHORT, R.E., ADAMS, D.C. 1988. Nutritional and hormonal interrelationships in beef cattle reproduction. Can. J. Anim. Sci., 68:29-39.

SILVA, D.J. 1990. Análises de alimentos: métodos químicos e biológicos. Imprensa Universitária, Viçosa, MG.165p.

SKLAN, D., MOALLEN, U., FOLMAN, Y. 1991. Effect of dietary calcium soaps of fatty on production and, reproductive responses in high producing lactating cows. J. Dairy Sci., 74:510-517.

SPICER, L.J., VERNON, R.K., TUCKER, W.B. et al. 1993. Effects of protected fat on plasma concentrations of cholesterol and progesterone in lactating dairy cows. Anim. Sci. Res. Reprod., P-933:175-178.

STABENFELDT, G.H., EWING, L.L., McDOLNALD, L.E. 1969. Peripheral plasma progesterone levels during the bovine oestrous cycle. J. Reprod. Fert., 19:433-442.
STAPLES, C.R., THATCHER, W.W., CLARK, J.H. 1990. Relationships between ovarian activity and energy status during the early postpartum period of high producing dairy cows. J. Dairy Sci., 73:938-947.

STEVENSON, J.S., CALL, E.P. 1983. Influence of early estrus, ovulation, and insemination on fertility in postpartum holstein cows. Theriogenology, 19:367-375.

TALAVERA, F., PARK, C.S., WILliAMS, G.L. 1985. Relationships among dietary lipid intake, serum cholesterol and ovarian function in holstein heifers. J. Anim. Sci., 60:10451051.

THOMAS, M.G., WILLIAMS, G.L. 1996. Metabolic hormone secretion and FSH-induced superovulatory responses of beef heifers fed dietary fat supplements containing predominantly satured or polyunsatured fatty acids. Theriogenology, 45:451-458.

VILLA-GODOY, A., HUGHES, T.L., EMERY, R.S. et al. 1988. Association between energy balance and luteal function in lactating dairy cows. J. Dairy Sci., 71:1063-1072.

WEHRMAN, M.E., WELSH, J.R., WILLIAMS, G.L. 1991. Diet-induced hyperlipidemia in cattle modifies the intrafollicular cholesterol environment, modulates ovarian follicular dynamics, and hastens the onset of postpartum luteal activity. Biol. Reprod., 45:514-522.

WILLIAMS, G.L. 1989. Modulation of luteal activity in postpartum beef cows through changes in dietary lipid. $J$. Anim. Sci., 67:785-793.

ZALESKY, D.D. DAY, M.L., GARCIA-WINDER, M. et al. 1984. Infuence of exposure to bulls on resumption of estrous cycles following parturition in beef cows. J. Anim. Sci., 59:1135-1139.

Recebido em: 08/03/99

Aceito em: 10/08/99 